\title{
The Ratio of Free Fatty Acids (FFAs) Divided by High- density Lipoprotein Cholesterol (HDL-C) is A Promising Pre-Treatment Biomarker for Predicting Worse Overall Survival (OS) of Neuroendocrine Tumours (NETs) in the Colorectum: A Retrospective Study
}

Bin Zhu

Fujian Medical University Union Hospital

Dan Wu

Fujian Medical University Union Hospital

Yuanyuan Yang

Fujian Medical University Union Hospital

Pingli Yu

Fujian Medical University Union Hospital

Haobo Huang

Fujian Medical University Union Hospital

Haiyan Cai

Fujian Medical University

Yingping Cao ( $\square$ caoyingping918@sina.com )

Fujian Medical University Union Hospital

\section{Research Article}

Keywords: Free fatty acid, HDL-C, Serum FFA/HDL-C Ratio, Overall survival, Colorectal neuroendocrine tumour.

Posted Date: February 15th, 2022

DOI: https://doi.org/10.21203/rs.3.rs-1227675/v2

License: (우 This work is licensed under a Creative Commons Attribution 4.0 International License. Read Full License 


\section{Abstract \\ Background}

free fatty acids (FFAs) and high-density lipoprotein cholesterol (HDL-C) were associated with various malignancy. However, whether FFA, HDL-C and FFA/HDL-C can play a potiential role in predicting patients' overall survival (OS) with colorectal neuroendocrine tumours (NETs) was unclear. Meanwhile, FFA/HDL-C has a superior prognosis ability was unknown, too. And our study aimed to better elucidate their influence on clinical outcomes.

\section{Methods}

One hundred patients with pathologically diagnosed colorectal NETs in 2011-2017 were enrolled, and the levels of FFA, HDL-C, low-density lipoprotein cholesterol (LDL-C), triglycerides (TGs), cholesterol (CHOL), apolipoprotein $\mathrm{A} 1$ (ApoA1) and apolipoprotein B (ApoB) between colorectal NET patients and healthy controls matched by age and sex were compared. In addition, the association of clinicopathological characteristics and follow-up data with FFA, HDL-C and FFA/HDL-C was analysed.

\section{Results}

FFA was overexpressed ( $0.55 \pm 0.23$ vs. $0.48 \pm 0.11, P=0.006)$, and HDL-C was underexpressed $(1.31 \pm 0.41$ vs. $1.41 \pm 0.29, P=0.046)$ in colorectal NETs. FFA $\geq 0.52 \mathrm{mmol} / \mathrm{L}$ predicted lymph node metastasis (LNM) $(P=0.015), H D L-C \leq 1.0 \mathrm{mmol} / \mathrm{L}$ predicted tumour size $\geq 2 \mathrm{~cm}(\mathrm{P}=0.017)$, and FFA/HDL-C $>0.75$ predicted tumour grade $(P=0.030)$, LNM $(P=0.014)$, and tumour size $(P=0.018)$. No significant association was found between FFA and tumour grade $(P=0.613)$ or $\mathrm{HDL}-\mathrm{C}$ and tumour grade $(\mathrm{P}=0.594)$ or $F F A$ and tumour size $(P=0.142)$ or HDL-C and LNM ( $P=0.443)$. FFA $\geq 0.52 \mathrm{mmol} / \mathrm{L}(P=0.014)$ and $\mathrm{HDL}-\mathrm{C} \leq 1.0 \mathrm{mmol} / \mathrm{L}$ predicted worse overall survival (OS) $(P=0.019)$. FFA/HDL-C predicted an even worse prognosis in terms of OS $(P<0.001)$.

\section{Conclusion}

FFA $\geq 0.52 \mathrm{mmol} / \mathrm{L} H D L-C \leq 1.0 \mathrm{mmol} / \mathrm{L}$ and FFA/HDL-C $>0.75$ were promising cut-off values in predicting LNM, tumour size and worse OS in colorectal NETs.

\section{Introduction}

Neuroendocrine tumours (NETs), previously described as "carcinoid tumours" in 1907, are heterogeneous neoplasms arising in secretory cells of the diffuse neuroendocrine system[1]. The Surveillance, Epidemiology and End Results (SEER) database indicates that the incidence of NETs has increased significantly, approximately 5 times, to $5.25 / 100.000$ cases/year; of these, colorectal NETs account for approximately $49.6 \%$ of primary NET sites in the digestive tract and have an incidence and prevalence inferior only to those 
of colorectal adenocarcinoma [2-4]. Although endoscopic mucosal resection, endoscopic submucosal dissection, surgery, radiation and chemotherapy have been used to treat localized and metastatic colorectal NETs, the 5-year survival rate of NETs with lymph node metastasis (LNM) or distant metastasis remains disappointing, with five-year overall survival rates of approximately $54-74 \%$ and $28-44.1 \%$, respectively $[5,6]$. According to the updated guidelines for colorectal NETs in 2016[7], tumour grade and size were only significant in univariate analysis, size remains a less than totally reliable discriminator of prognosis, and factors predicting LNM remain more researched. Currently, more reliable serological indicators that can predict prognosis in colorectal NETs have not been deeply demonstrated.

Free fatty acids (FFAs) are triacylglycerol (TG) precursors that are needed to replenish TG stores in adipose, liver and muscle tissue through esterification; when hepatic glycogen is low and muscles need energy, the TGs in adipose tissue are broken down into FFAs for energy[8]. FFAs are overexpressed in colorectal patients [9], and abnormal fatty acid metabolism is associated with tumour cell metastasis in various cancers, including colorectal cancer $[10,11]$. High-density lipoprotein $(H D L)$ is responsible for the reverse transport of cholesterol from peripheral cells to the liver, and associated apolipoproteins and enzymes can also exert antioxidant, anti-inflammatory, antiangiogenic, antiapoptotic and immunomodulatory activities, resulting in overall antitumorigenic effects[12]. HDL-C is involved in poor disease-free survival (DFS) and overall survival (OS) in colorectal cancer, and very low levels of $\mathrm{HDL}-\mathrm{C}(<30 \mathrm{mg} / \mathrm{dL})$ in women are significantly associated with cancer mortality[13, 14].

In clinical work, we found that in some cancer patients, traditional tumour markers such as carcinoembryonic antigen (CEA) and carbohydrate antigen 19-9 (CA19-9) were elevated tens of times compared with the upper limit of the healthy reference, while the standard deviations of lipid metabolism biomarkers in patients and controls were relatively similar. Is there a lipid biomarker with an advantage similar to that of traditional tumour markers? In addition, evidence for the prognostic ability of FFAs, HDL-C, and FFA/HDL-C and their relationship in colorectal NETs has never been reported. We designed this study to evaluate the prognostic ability of FFA, HDL-C and FFA/HDL-C in patients with colorectal NETs, compare FFA with HDL-C and analyse their combination.

\section{Materials And Methods}

\section{Patients}

Between 2011 and 2017, a total of 100 consecutive patients who received treatment for colorectal NETs in our hospital were enrolled. We constructed a database of retrospectively collected data from patient medical records, including clinical characteristics, pathological reports and survival during the follow-up period.

For local excision procedures, such as endoscopic submucosal dissection (ESD) and transanal excision (TAE), LNM was evaluated through computed tomography (CT) or magnetic resonance imaging (MRI) before the treatment and during the follow-up periods. The diagnosis of a metastatic lymph node was based on the following criteria: 1) Size criteria: short axis diameter greater than $8 \mathrm{~mm}$ for round lymph nodes and greater than $10 \mathrm{~mm}$ for ovoid lymph nodes; and 2) Morphological abnormalities: irregular contour and margin, unclear border, heterogeneous internal echoes or signal intensity. The tumour diameter refers to the longest 
diameter of the tumour according to pathology reports. For patients with distant metastases, tumour diameter was determined by endoscopic findings before treatment. The baseline characteristics of patients with colorectal NETs are listed in Table 1. 
Table 1

Clinical and Histopathological Characteristics of Colorectal NETs ( $N=100)$

\begin{tabular}{|c|c|}
\hline Variables & $\mathbf{N}(\%)$ \\
\hline \multicolumn{2}{|l|}{ Age } \\
\hline $21-30$ & $7(7 \%)$ \\
\hline $31-40$ & $11(11 \%)$ \\
\hline $41-50$ & $20(20 \%)$ \\
\hline $51-60$ & $28(28 \%)$ \\
\hline 61-70 & $24(24 \%)$ \\
\hline$\geq 71$ & $10(10 \%)$ \\
\hline \multicolumn{2}{|l|}{ Gender } \\
\hline Male & $65(65 \%)$ \\
\hline Female & $35(35 \%)$ \\
\hline \multicolumn{2}{|l|}{ BMI } \\
\hline$<23$ & $41(41 \%)$ \\
\hline$\geq 23$ & $59(59 \%)$ \\
\hline \multicolumn{2}{|l|}{ Tumour location } \\
\hline Rectum & $28(28 \%)$ \\
\hline Colon & $72(72 \%)$ \\
\hline \multicolumn{2}{|l|}{ Treatment } \\
\hline ESD & $14(14 \%)$ \\
\hline Transanal excision & $26(26 \%)$ \\
\hline Radical resection & $38(38 \%)$ \\
\hline Multivisceral resection & $8(8 \%)$ \\
\hline Palliative resection & $7(7 \%)$ \\
\hline Systemic treatment & $7(7 \%)$ \\
\hline \multicolumn{2}{|l|}{ Tumour diameter (cm) } \\
\hline$<2$ & $45(45.5)$ \\
\hline$\geq 2$ & $54(54.5 \%)$ \\
\hline
\end{tabular}

Abbreviation: NET: neuroendocrine tumour; BMI, body mass index; ESD:endoscopic submucosal dissection; Syn: synaptophysin; CgA: chromogranin A; CD 56: cluster of differentiation 56; CK: cytokeratin. 


\begin{tabular}{|c|c|}
\hline Variables & $\mathbf{N}(\%)$ \\
\hline \multicolumn{2}{|c|}{ Lymph node metastasis } \\
\hline Negative & $45(46.9 \%)$ \\
\hline Positive & $51(53.1 \%)$ \\
\hline \multicolumn{2}{|c|}{ Tumour grade } \\
\hline G1 & $57(57 \%)$ \\
\hline G2 & $14(14 \%)$ \\
\hline G3 & $29(29 \%)$ \\
\hline \multicolumn{2}{|l|}{ Syn } \\
\hline- & $5(5.4 \%)$ \\
\hline \pm & $15(16.3 \%)$ \\
\hline+ & $72(78.3 \%)$ \\
\hline \multicolumn{2}{|l|}{$\mathrm{CgA}$} \\
\hline- & $50(56.8 \%)$ \\
\hline \pm & $24(27.3 \%)$ \\
\hline+ & $14(15.9 \%)$ \\
\hline \multicolumn{2}{|l|}{ CD56 } \\
\hline- & $19(22.6 \%)$ \\
\hline \pm & $10(11.9 \%)$ \\
\hline+ & $55(65.5 \%)$ \\
\hline \multicolumn{2}{|l|}{ CK } \\
\hline- & $2(3.0 \%)$ \\
\hline \pm & $3(4.5 \%)$ \\
\hline+ & $61(92.4 \%)$ \\
\hline
\end{tabular}

The healthy controls were matched by age and sex one by one to patients with colorectal NETs.

\section{Pathological Diagnosis}

According to the 2010 World Health Organization classification[15], tumour grade was defined numerically, in which low-grade (grade 1 [G1]) tumours have a mitotic rate from 0 to 1 per 10 high-power fields (HPFs) or a Ki-67 index from $0-2 \%$, intermediate-grade (G2) tumours have a mitotic rate from 2 to 20 per $10 \mathrm{HPFs}$ or a Ki- 
67 index from 3-20\%, and high-grade (G3) tumours have a mitotic rate greater than 20 per $10 \mathrm{HPFs}$ or a Ki-67 index greater than $20 \%[16]$. The expression levels of chromogranin $A(\mathrm{CgA})(\mathrm{N}=88)$, synaptophysin (Syn) $(\mathrm{N}=92)$, cluster of differentiation $56(\mathrm{CD} 56)(\mathrm{N}=84)$ and cytokeratin $(\mathrm{CK})(\mathrm{N}=66)$ were scored according to the percentage of positive cells and the intensity of cell staining; no positive cells or negative cell staining intensity was labelled (-); a small number of positive cells or weakly positive cell staining intensity was labelled ( \pm ); and the majority of positive cells or strongly positive cell staining intensity was labelled (+).

\section{Laboratory testing}

FFAs, HDL-C,TGs, cholesterol (CHOL), low-density lipoprotein cholesterol (LDL-C), apolipoprotein A1 (ApoA1)and apolipoprotein B (ApoB) were measured routinely with a Cobas 8000 automatic biochemical analyser (Roche, Diagnostics, Basel, Switzerland) according to the manufacturer's instructions. The enzyme endpoint method was applied to measure the levels of FFAs, TGs, CHOL, HDL-C and LDL-C. An immunoturbidimetric assay was applied to measure the levels of ApoA1 and ApoB. The FFAs that we detected were nonesterified fatty acids (Wako Pure Chemical Corporation, Japan); in addition, TG, CHOL, HDLC LDL-C ApoA1 and ApoB were detected by original reagents (Roche, Diagnostics, Indianapolis, USA).

\section{Inclusion Criteria}

Patients who were treated in our centre for localized and metastatic colorectal NETs by pathological diagnosis from 2010 to 2017.

\section{Exclusion Criteria}

1.Colorectal NETs combined with other malignancies. 2. Insufficient clinical information or in appropriate pathology reports from outside hospitals.

\section{Statistical Analysis}

Statistical analysis was performed using SPSS for Mac, version 21.0 or GraphPad Prism version 8.0. Continuous data are described as the means \pm SDs in this study. The relationship between pathological characteristics and FFAs as well as HDL-C was assessed using Pearson's $\chi^{2}$ or Fisher's exact test. Overall survival (OS) was analysed with the Kaplan-Meier method. Univariable and multivariable analyses for survival outcomes were conducted using the Cox proportional hazards model. Statistical significance was accepted for $p$ values $<0.05$.

\section{Results}

In this study, data from 100 colorectal NET patients and 100 control persons matched by sex and age were analysed. The age of the patients was $54.1 \pm 13.7$ years, and the male: female ratio was $65(65.0 \%): 35$ (35.0\%). The numbers of grade G1, G2, and G3 NETs were 57(57.0\%), 14 (14.0\%) and 29 (29.0\%), respectively. Of the 100 patients, $40(40.0 \%)$ patients were resected locally, 14(14\%) by ESD and $26(26 \%)$ by transanal excision. In addition, $53(53.0 \%)$ NETs were surgically resected, including $38(38.0 \%)$ radical resections, 8 (8.0\%) multivisceral resections and $7(7.0 \%)$ palliative resections due to distant metastasis. The remaining 7 $(7.0 \%)$ patients were treated by systemic treatment due to distant metastasis. The most commonly used chemotherapeutic regimen in our centre was the EP regimen (etoposide and cisplatin) as the first-line 
chemotherapy, and the second-line chemotherapy was variable and included the XELOX regimen (oxaliplatin and capecitabine); the FOLFOX regimen (oxaliplatin, calciumfolate and 5-FU); and everolimus, temozolomide, and tegafur/gimeracil/oteracil and their combinations. The tumour diameter was less than $2 \mathrm{~cm}$ in 45(45.5\%) patients, and LNM was found in 51(53.1\%) patients. The clinical and histopathological characteristics are summarized in Table 1.

The pre-treatment levels of FFAs $(0.55 \pm 0.23$ vs. $0.48 \pm 0.11, \mathrm{P}=0.006), \mathrm{CHOL}(4.84 \pm 0.87$ vs. $4.56 \pm 0.66$, $P=0.011), A p o B(0.96 \pm 0.26$ vs. $0.89 \pm 0.16, P=0.030)$ and $F F A / H D L-C(0.51 \pm 0.61$ vs. $0.38 \pm 0.11, P=0.027)$ in patients with colorectal NETs were higher than those in controls. The pre-treatment levels of HDL-C ( $1.31 \pm 0.41$ vs. $1.41 \pm 0.29, P=0.046)$ and $A p o A 1(1.18 \pm 0.43$ vs. $1.54 \pm 0.17, P<0.001)$ were lower in patients than in controls. There was no significant difference in the levels of TGs $(1.33 \pm 0.80$ vs. $1.24 \pm 0.48, P=0.346)$ or LDL-C ( $3.10 \pm 0.78$ vs. $2.98 \pm 0.65, \mathrm{P}=0.251)$ between patients and controls. The data are illustrated in Figure 1.

The clinical and laboratory parameters, as well as the comparison between the high FFA and low FFA groups, are shown in Table 2; similar parameters and comparisons with regard to HDL-C are also shown. Pearson's $\chi^{2}$ test showed significant associations between FFA and lymph node metastasis $\left(\chi^{2}=5.964, P=0.015\right)$ and between HDL-C and tumour size $\left(X^{2}=5.647, P=0.017\right)$. However, no significant association was found between FFA and tumour size ( $P=0.142)$ or between HDL-C and lymph node metastasis $(P=0.443)$. There were significant associations of FFA/HDL-C with tumour grade $\left(X^{2}=4.734, P=0.030\right)$, lymph node metastasis $\left(X^{2}=\right.$ $6.032, P=0.014)$, and tumour size $\left(X^{2}=5.583, P=0.018\right)$. Neither FFA, HDL-C nor FFA/HDL-C was found to be significantly associated with sex $\left(P_{F F A}=0.542, P_{H D L-C}=0.157, P_{F F A / H D L-C}=0.297\right)$, age $\left(P_{F F A}=0.096\right.$, $\left.\mathrm{P}_{\mathrm{HDL}-\mathrm{C}}=0.940, \mathrm{P}_{\mathrm{FFA} / \mathrm{HDL}-\mathrm{C}}=0.331\right), \mathrm{BMI}\left(\mathrm{P}_{\mathrm{FFA}}=0.841, \mathrm{P}_{\mathrm{HDL}-\mathrm{C}}=0.799, \mathrm{P}_{\mathrm{FFA} / \mathrm{HDL}-\mathrm{C}}=1.0\right)$, tumour grade $\left(P_{F F A}=0.613, P_{H D L-C}=0.594\right)$, Syn $\left(P_{F F A}=0.926, P_{H D L-C}=0.411, P_{F F A / H D L-C}=0.854\right), C g A\left(P_{F F A}=0.546\right.$, $\left.P_{H D L-C}=0.214, P_{F F A / H D L-C}=0.534\right), C D 56\left(P_{F F A}=0.460, P_{H D L-C}=0.662, P_{F F A / H D L-C}=0.254\right)$, or $C K\left(P_{F F A}=0.321\right.$, $P_{\text {HDL-C }}=0.083, P_{\text {FFA/HDL-C }}=0.071$ ).

Table 2 The serological levels of FFAs and HDL-C in colorectal NETs with different pathological characteristics. 
FFA N (\%)

Parameters
HDL-C N (\%)

$P$ value

$\geq 0.52$

$\mathrm{mmol} / \mathrm{L}$
$\mathrm{X}^{2}$ value

$\leq 1.0$

$\mathrm{mmol} / \mathrm{L}$
$P$ value

$>1.0$

$\mathrm{mmol} / \mathrm{L}$
FFA/HDL-CN (\%)

$x^{2} \quad P$ value value $>0.75 \leq 0.75$

\section{Gender}

Male

Female

$32(32 \%)$

$33(33 \%)$

$15(15 \%)$
$4(4 \%)$
2.006

$50(50 \%)$

8(8\%) 57(57\%)

$15(15 \%)$

$20(20 \%)$

0.371

$0.542^{\mathrm{a}}$

$31(31 \%)$

$2(2 \%) \quad 33(33 \%)$

$0.157^{a}$

$1.088 \quad 0.297^{b}$

\section{Age \\ $<65$ \\ $\geq 65$}

$38(38 \%)$

$35(35 \%)$

$14(14 \%)$
$5(5 \%)$

$59(59 \%)$

$6(6 \%) \quad 67(67 \%)$

2.773

18(18\%)

0.006

$22(22 \%)$

$4(4 \%) \quad 23(23 \%)$

$0.096^{a}$

$\begin{array}{ll}23(23 \%) & 27(27 \%) \\ 24(24 \%) & 26(26 \%) \\ 0.4 & 0.841^{a}\end{array}$

$\begin{array}{llll}9(9 \%) & 41(41 \%) & 5(5 \%) & 45(45 \%) \\ 10(10 \%) & 40(40 \%) & 5(5 \%) & 45(45 \%) \\ 0.065 & 0.799^{a} & 0 & 1.0^{a}\end{array}$

\section{Tumour grade}

\begin{tabular}{lllllll} 
G1 & $27(27 \%)$ & $30(30 \%)$ & $9(9 \%)$ & $48(48 \%)$ & $2(2 \%)$ & $55(55 \%)$ \\
\hline G2 & $5(5 \%)$ & $9(9 \%)$ & $3(3 \%)$ & $11(11 \%)$ & $3(3 \%)$ & $11(11 \%)$ \\
G3 & $15(15 \%)$ & $14(14 \%)$ & $7(7 \%)$ & $22(22 \%)$ & $5(5 \%)$ & $24(24 \%)$ \\
\hline & 0.979 & $0.613^{\mathrm{a}}$ & 1.131 & $0.594^{\mathrm{b}}$ & 4.734 & $0.030^{\mathrm{b}}$
\end{tabular}

\section{Lymph node \\ metastasis}

\begin{tabular}{lllllll} 
Negative & $28(29.2 \%)$ & $17(17.7 \%)$ & $7(7.3 \%)$ & $38(39.6 \%)$ & $1(1.0 \%)$ & $44(45.8 \%)$ \\
\hline Positive & $19(19.8 \%)$ & $32(33.3 \%)$ & $12(12.5 \%)$ & $39(40.6 \%)$ & $9(9.4 \%)$ & $42(43.8)$ \\
\hline & 5.964 & $0.015^{a}$ & 0.958 & $0.443^{\mathrm{a}}$ & 6.032 & $\mathbf{0 . 0 1 4 ^ { \mathrm { b } }}$
\end{tabular}

\section{Tumour Size}

\begin{tabular}{lllllll}
$<2 \mathrm{~cm}$ & $25(25.3 \%)$ & $20(20.2 \%)$ & $4(4.0 \%)$ & $41(41.4 \%)$ & $1(1.0 \%)$ & $44(44.4 \%)$ \\
$\geq 2 \mathrm{~cm}$ & $22(22.2 \%)$ & $32(32.3 \%)$ & $15(15.2 \%)$ & $39(39.4 \%)$ & $9(9.1 \%)$ & $45(45.5 \%)$ \\
\hline 2.16 & $0.142^{\mathrm{a}}$ & 5.647 & $0.017^{\mathrm{a}}$ & 5.583 & $\mathbf{0 . 0 1 8 ^ { \mathrm { b } }}$
\end{tabular}


Syn

\begin{tabular}{lllllll}
- & $2(2.2 \%)$ & $3(3.3 \%)$ & $2(2.2 \%)$ & $3(3.3 \%)$ & $0(0 \%)$ & $5(5.4 \%)$ \\
\hline & $8(8.7 \%)$ & $7(7.6 \%)$ & $2(2.2 \%)$ & $13(14.1 \%)$ & $2(2.2 \%)$ & $13(14.1 \%)$ \\
\hline & $34(37.0 \%)$ & $38(41.2 \%)$ & $14(15.2 \%)$ & $58(63.0 \%)$ & $8(8.7 \%)$ & $64(69.6 \%)$ \\
\hline & 0.414 & $0.926^{\mathrm{b}}$ & 1.809 & $0.411^{\mathrm{b}}$ & 0.034 & $0.854^{\mathrm{b}}$
\end{tabular}

CgA

\begin{tabular}{lllllll}
- & $24(27.3)$ & $26(29.5 \%)$ & $13(14.8 \%)$ & $37(42 \%)$ & $7(8.0 \%)$ & $43(48.9 \%)$ \\
\hline \pm & $13(14.8 \%)$ & $11(12.5 \%)$ & $2(2.3 \%)$ & $22(25 \%)$ & $2(2.3 \%)$ & $22(25.0 \%)$ \\
\hline+ & $5(5.7 \%)$ & $9(10.2 \%)$ & $3(3.4 \%)$ & $11(12.5 \%)$ & $1(1.1 \%)$ & $13(14.8 \%)$ \\
\hline & 1.21 & $0.546^{\mathrm{a}}$ & 3.138 & $0.214^{\mathrm{b}}$ & 0.387 & $0.534^{\mathrm{b}}$
\end{tabular}

\section{CD56}

\begin{tabular}{lllllll}
- & $7(8.3 \%)$ & $12(14.3 \%)$ & $5(6 \%)$ & $14(16.7 \%)$ & $4(4.7 \%)$ & $15(17.9 \%)$ \\
\hline \pm & $6(7.1 \%)$ & $4(4.8 \%)$ & $1(1.2 \%)$ & $9(10.7 \%)$ & $1(1.2 \%)$ & $9(10.7 \%)$ \\
\hline+ & $27(32.1 \%)$ & $28(33.3 \%)$ & $11(13.1 \%)$ & $44(52.4 \%)$ & $5(6.0 \%)$ & $50(59.5 \%)$ \\
\hline & 1.54 & $0.46^{\mathrm{b}}$ & 0.969 & $0.662^{\mathrm{b}}$ & 1.302 & $0.254^{\mathrm{b}}$ \\
\hline $\mathbf{C K}$ & & & & & & \\
\hline $\mathbf{I}$ & $0(0 \%)$ & $2(3 \%)$ & $1(1.5 \%)$ & $1(1.5 \%)$ & $1(1.5 \%)$ & $1(1.5 \%)$ \\
\hline+ & $1(1.5 \%)$ & $2(3 \%)$ & $2(3 \%)$ & $1(1.5 \%)$ & $1(1.5 \%)$ & $2(3.0 \%)$ \\
\hline & $33(50 \%)$ & $28(42.4 \%)$ & $11(16.7 \%)$ & $50(75.8 \%)$ & $5(7.6 \%)$ & $56(84.8)$ \\
\hline
\end{tabular}

a: It was detected by Pearson's chi-squared test; b:It was detected by Fisher's exact test. Abbreviations: NET: neuroendocrine tumour; BMl, body mass index; Syn: synaptophysin; CgA: chromogranin A; CD56: cluster of differentiation 56; CK: cytokeratin. G1:a mitotic rate from 0 to 1 per 10high-power fields (HPFs) or a Ki-67 index from $0 \%$ to $2 \%$; G2: a mitotic rate from 2 to 20 per $10 \mathrm{HPFs}$ or a $\mathrm{Ki}-67$ index from $3 \%$ to $20 \%$; G3: a mitotic rate greater than 20 per $10 \mathrm{HPFs}$ or a Ki-67 index greater than $20 \%$.

Univariable and multivariable analyses were performed to analyse the potential prognostic factors for survival. The median follow-up period of this cohort was 70 months (range: 1-130 months). Univariable analysis showed that higher serum FFA levels $(\geq 0.52 \mathrm{mmol} / \mathrm{l})(P=0.014)$, lower serum HDL-C levels $(\leq 1.0$ $\mathrm{mmol} / \mathrm{L})(P=0.022)$, higher serum FFA/HDL-C levels $(>0.75)(P<0.001)$, the presence of tumour grades $\mathrm{G} 2$ $(P=0.003)$ and $G 3(P<0.001)$, positive lymph node metastasis $(P<0.001)$, tumour size larger than $2 \mathrm{~cm}$ $(P<0.001)$, and age older than 65 years $(P=0.046)$ were significantly associated with shorter OS. Multivariable 
analysis showed that tumour grade $G 3$ and positive lymph node metastasis in the FFA group $\left(P_{G 3}=0.013\right.$, $\left.P_{L N M}=0.048\right)$, HDL-C group $\left(P_{G 3}=0.017, P_{L N M}=0.016\right)$ and FFA/HDL-C group $\left(P_{G 3}=0.021, P_{L N M}=0.037\right)$ were significantly associated with shorter OS. There was no significant association between shorter OS and tumour size/FFA/HDL-C/FFA divided by HDL-C. The hazard ratios (HRs) and corresponding $95 \%$ confidence intervals are shown in Table 3. 
Table 3

Univariable and multivariable analysis of prognostic factors for OS in patients with colorectal NETs.

\begin{tabular}{|c|c|c|c|c|c|c|c|c|}
\hline \multirow[t]{2}{*}{ Parameters } & \multicolumn{2}{|c|}{ Univariate analysis } & \multicolumn{2}{|l|}{$\begin{array}{l}\text { Multivariate } \\
\text { analysis } \\
\text { (FFA) }\end{array}$} & \multicolumn{2}{|l|}{$\begin{array}{l}\text { Multivariate } \\
\text { analysis } \\
\text { (HDL-C) }\end{array}$} & \multicolumn{2}{|c|}{$\begin{array}{l}\text { Multivariate } \\
\text { analysis } \\
\text { (FFA/HDL-C) }\end{array}$} \\
\hline & $\begin{array}{l}\mathrm{HR} \\
(95 \% \mathrm{Cl})\end{array}$ & $\begin{array}{l}\mathrm{P} \\
\text { value }\end{array}$ & $\begin{array}{l}\mathrm{HR} \\
(95 \% \mathrm{Cl})\end{array}$ & Pvalue & $\begin{array}{l}\mathrm{HR} \\
(95 \% \mathrm{Cl})\end{array}$ & Pvalue & $\begin{array}{l}\mathrm{HR} \\
(95 \% \mathrm{Cl})\end{array}$ & Pvalue \\
\hline Gender & 1.325 & 0.355 & & & & & & \\
\hline $\begin{array}{l}\text { (male vs. } \\
\text { female) }\end{array}$ & $\begin{array}{l}(0.729- \\
2.408)\end{array}$ & & & & & & & \\
\hline \multirow{2}{*}{$\begin{array}{l}\text { Age }(<65 \\
\text { vs. } \geq 65)\end{array}$} & 1.863 & 0.046 & & & & & & \\
\hline & $\begin{array}{l}(1.011- \\
3.432)\end{array}$ & & & & & & & \\
\hline \multirow{2}{*}{$\begin{array}{l}\text { BMI (<23 } \\
\text { vs. } \geq 23)\end{array}$} & 1.354 & 0.313 & & & & & & \\
\hline & $\begin{array}{l}(0.752- \\
2.438)\end{array}$ & & & & & & & \\
\hline \multirow{2}{*}{$\begin{array}{l}\text { Tumour } \\
\text { Grade }\end{array}$} & 2.295 & $<0.001$ & & & & & & \\
\hline & $\begin{array}{l}(1.652- \\
3.19)\end{array}$ & & & & & & & \\
\hline G1 & Reference & & Reference & 0.044 & Reference & 0.056 & Reference & 0.069 \\
\hline \multirow[t]{2}{*}{ G2 } & 3.639 & 0.003 & 1.986 & 0.126 & 1.837 & 0.183 & 1.826 & 0.187 \\
\hline & $\begin{array}{l}(1.554- \\
8.524)\end{array}$ & & $\begin{array}{l}(0.825- \\
4.779)\end{array}$ & & $\begin{array}{l}(0.750- \\
4.498)\end{array}$ & & $\begin{array}{l}(0.746- \\
4.469)\end{array}$ & \\
\hline \multirow[t]{2}{*}{ G3 } & 5.487 & $<0.001$ & 2.518 & 0.013 & 2.465 & 0.017 & 2.370 & 0.021 \\
\hline & $\begin{array}{l}(2.756- \\
10.926)\end{array}$ & & $\begin{array}{l}(1.216- \\
5.216)\end{array}$ & & $\begin{array}{l}(1.178- \\
5.159)\end{array}$ & & $\begin{array}{l}(1.137- \\
4.938)\end{array}$ & \\
\hline LNM & 10.545 & $<0.001$ & 3.081 & 0.048 & 3.944 & 0.016 & 3.296 & 0.037 \\
\hline $\begin{array}{l}\text { (Neg vs. } \\
\text { Pos) }\end{array}$ & $\begin{array}{l}(4.433- \\
25.082)\end{array}$ & & $\begin{array}{l}(1.008- \\
9.415)\end{array}$ & & $\begin{array}{l}(1.295- \\
12.015)\end{array}$ & & $\begin{array}{l}(1.078- \\
10.083)\end{array}$ & \\
\hline
\end{tabular}

Abbreviations: BMI, body mass index;LNM: lymph node metastasis;HR: hazard ratio. 


\begin{tabular}{|c|c|c|c|c|c|c|c|c|}
\hline \multirow[t]{2}{*}{ Parameters } & \multicolumn{2}{|c|}{ Univariate analysis } & \multicolumn{2}{|c|}{$\begin{array}{l}\text { Multivariate } \\
\text { analysis } \\
\text { (FFA) }\end{array}$} & \multicolumn{2}{|c|}{$\begin{array}{l}\text { Multivariate } \\
\text { analysis } \\
(\text { HDL-C) }\end{array}$} & \multicolumn{2}{|c|}{$\begin{array}{l}\text { Multivariate } \\
\text { analysis } \\
\text { (FFA/HDL-C) }\end{array}$} \\
\hline & $\begin{array}{l}\mathrm{HR} \\
(95 \% \mathrm{Cl})\end{array}$ & $\begin{array}{l}P \\
\text { value }\end{array}$ & $\begin{array}{l}\mathrm{HR} \\
(95 \% \mathrm{Cl})\end{array}$ & Pvalue & $\begin{array}{l}\mathrm{HR} \\
(95 \% \mathrm{Cl})\end{array}$ & Pvalue & $\begin{array}{l}\mathrm{HR} \\
(95 \% \mathrm{Cl})\end{array}$ & Pvalue \\
\hline $\begin{array}{l}\text { Tumour } \\
\text { Size }\end{array}$ & $\begin{array}{l}14.519 \\
(5.166- \\
40.802)\end{array}$ & $<0.001$ & $\begin{array}{l}3.684 \\
(0.965- \\
14.071)\end{array}$ & 0.056 & $\begin{array}{l}2.950 \\
(0.752- \\
11.574)\end{array}$ & 0.121 & $\begin{array}{l}3.438 \\
(0.848- \\
13.933)\end{array}$ & 0.084 \\
\hline \multicolumn{9}{|l|}{$\begin{array}{l}(<2 \text { vs. } \geq 2 \\
\mathrm{cm})\end{array}$} \\
\hline FFA & 1.958 & 0.014 & 1.804 & 0.067 & & & & \\
\hline $\begin{array}{l}\text { (high vs. } \\
\text { normal) }\end{array}$ & $\begin{array}{l}(1.063- \\
3.608)\end{array}$ & & $\begin{array}{l}(0.960- \\
3.388)\end{array}$ & & & & & \\
\hline HDL-C & 0.484 & 0.022 & & & 0.632 & 0.2 & & \\
\hline $\begin{array}{l}\text { (high vs. } \\
\text { normal) }\end{array}$ & $\begin{array}{l}(0.254- \\
0.922)\end{array}$ & & & & $\begin{array}{l}(0.314- \\
1.275)\end{array}$ & & & \\
\hline FFA/HDL-C & 3.630 & $<0.001$ & & & & & 2.015 & 0.083 \\
\hline $\begin{array}{l}\text { (high vs. } \\
\text { normal) }\end{array}$ & $\begin{array}{l}(1.685- \\
7.819)\end{array}$ & & & & & & $\begin{array}{l}(0.913- \\
4.445)\end{array}$ & \\
\hline
\end{tabular}

Kaplan-Meier survival curves were plotted to analyse the different OS periods in colorectal NETs. The logrank (Mantel-Cox) test showed that patients with high tumour grade $\left(X^{2}=28.69, P<0.001\right)$, positive lymph node metastasis $\left(X^{2}=41.43, P<0.001\right)$, larger tumours $\left(X^{2}=44.36, P<0.001\right)$, higher FFA level $\left(X^{2}=6.016\right.$, $P=0.014)$, lower HDL-C level $\left(X^{2}=5.488, P=0.019\right)$, both double-expressed patients (higher FFA and lower HDLC) $\left(X^{2}=4.818, P=0.028\right)$ and higher FFA/HDL-C level $\left(X^{2}=12.528, P<0.001\right)$ had worse overall survival. The 5year survival of FFA/HDL-C in different score groups $(0.75-1,0.5-0.75,0.25-0.5,0-0.25)$ decreased gradually $(P=0.004)$. The Kaplan-Meier curves are drawn in Figure 2.

We found that 5-year survival declined from grades G1 to G3 $(78.6 \%, 42.9 \%$ and $27.6 \%)$ and was lower for LNM-positive patients ( $29.4 \%$ vs. $88.8 \%$ ) and those with larger tumours (31.5\% vs. $89.1 \%)$, a high level of FFAs ( $50.9 \%$ vs. $70.2 \%$ ), a low level of HDL-C (47.4\% vs. $63.0 \%)$, double-expressed patients (high FFAs with low HDL-C) (40.0\% vs. $62.2 \%)$ and FFAs/HDL-C (30.0\% vs. $63.3 \%)$. In addition, the median survival also 
declined from grades $\mathrm{G} 1$ to $\mathrm{G} 3(105.1 \pm 6.2,59.6 \pm 12.1$ and $42.0 \pm 7.8)$ and was poorer for patients with $\mathrm{LNM}$ positivity (43.6 \pm 6.2 vs. $118.0 \pm 4.6)$, larger tumour size ( $46.2 \pm 6.2$ vs. $121.2 \pm 4.2)$, high level of FFA $(67.8 \pm 7.3$ vs. $94.7 \pm 7.3)$, low level of HDL-C (53.8 \pm 9.7 vs. $87.1 \pm 6.0)$, high double-expressed patients (high FFA with low HDLC) $(45.9 \pm 14.3$ vs. $85.2 \pm 5.7)$ and FFA/HDL-C (35.3 \pm 13.7 vs. $86.7 \pm 5.5)$. The data are shown in Table 4.

Table 4

Five-year survival and median survival months atdifferent levels of FFAs, HDL$\mathrm{C}, \mathrm{FFA} / \mathrm{HDL}-\mathrm{C}$, tumour grade, LNM, tumour size and FFA in combination with HDL-C.

\begin{tabular}{|c|c|c|c|}
\hline Parameters & $\begin{array}{l}\text { 5-year survival } \\
\text { (\%) }\end{array}$ & $\begin{array}{l}\text { Median survivalmonths } \\
\text { (Mean } \pm \text { SD) }\end{array}$ & $P$ value \\
\hline \multicolumn{4}{|l|}{ Tumour Grade } \\
\hline G1 & 78.6 & $105.1 \pm 6.2$ & \\
\hline G2 & 42.9 & $59.6 \pm 12.1$ & \\
\hline G3 & 27.6 & $42.0 \pm 7.8$ & $<0.001$ \\
\hline \multicolumn{4}{|l|}{ Lymphnode metastasis } \\
\hline Negative & 88.8 & $118.0 \pm 4.6$ & \\
\hline Positive & 29.4 & $43.6 \pm 6.2$ & $<0.001$ \\
\hline \multicolumn{4}{|l|}{ Tumour Size } \\
\hline$<2 \mathrm{~cm}$ & 89.1 & $121.2 \pm 4.2$ & \\
\hline$\geq 2 \mathrm{~cm}$ & 31.5 & $46.2 \pm 6.2$ & $<0.001$ \\
\hline \multicolumn{4}{|l|}{ FFA } \\
\hline$<0.52 \mathrm{mmol} / \mathrm{L}$ & 70.2 & $94.7 \pm 7.3$ & \\
\hline$\geq 0.52 \mathrm{mmol} / \mathrm{L}$ & 50.9 & $67.8 \pm 7.3$ & 0.027 \\
\hline \multicolumn{4}{|l|}{ HDL-C } \\
\hline$>1.0 \mathrm{mmol} / \mathrm{L}$ & 63.0 & $87.1 \pm 6.0$ & \\
\hline$\leq 1.0 \mathrm{mmol} / \mathrm{L}$ & 47.4 & $53.8 \pm 9.7$ & 0.024 \\
\hline \multicolumn{4}{|l|}{ Doubleexpressed patients } \\
\hline Others & 62.2 & $85.2 \pm 5.7$ & \\
\hline $\mathrm{FFA} \geq 0.52+\mathrm{HDL}-\mathrm{C} \leq 1.0$ & 40.0 & $45.9 \pm 14.3$ & 0.026 \\
\hline \multicolumn{4}{|l|}{ FFA/HDL-C } \\
\hline$\leq 0.75$ & 63.3 & $86.7 \pm 5.5$ & \\
\hline$>0.75$ & 30.0 & $35.3 \pm 13.7$ & $<0.001$ \\
\hline
\end{tabular}




\section{Discussion}

From a follow-up period of up to 130 months, we collected consecutive colorectal NET patients by pathological diagnosis from 2011-2017 and found that FFA $\geq 0.52 \mathrm{mml} / \mathrm{L}$ can be considered a cut-off point for predicting LNM and poor OS, HDL-C $\leq 1.0 \mathrm{mmol} / \mathrm{L}$ can be considered another cut-off point for predicting tumour size $\geq 2 \mathrm{~cm}$ and poor OS, and FFA/HDL-C $>0.75$ predicted tumour grade, LNM, tumour size and even worser OS in our study.

According to the updated guidelines for colorectal NETs in 2016[7], tumour grade, size, symptoms, and treatment modality were only significant in univariate analysis, and stage was the strongest predictor of survival in multivariate analysis; on the one hand, tumour size and depth can predict LNM; on the other hand, size remains a less than totally reliable discriminator of prognosis, and factors predicting LNM remain more researched. Currently, whether serological indicators can predict prognosis in colorectal NETs has not been deeply demonstrated. In our study, we identified a new biomarker of FFA/HDL-C that has a promising ability to predict prognosis as well as tumour grade, size and LNM. In addition, the predictions of FFA, HDL-C and FFA/HDL-C were relatively independent and complementary, and the OS prognosis of FFA/HDL-C was even worse than that of FFA or HDL-C. Combined detection of FFA and HDL-C is a promising biomarker in colorectal NETs, and it may benefit new guidelines in the future.

In clinical work, we found that in some cancer patients, traditional tumour markers such as carcinoembryonic antigen (CEA) and carbohydrate antigen 19-9 (CA19-9) were elevated tens of times compared with the upper limit of the healthy reference, while the standard deviations of lipid metabolism biomarkers in patients and controls were relatively similar. In our study, we found that the FFA/HDL-C ratio of cancer patients has more scatter points than other lipid metabolism biomarkers, as shown in Figure 1. These scatter points, which have similar changes in FFA/HDL-C to CEA and CA19-9, account for almost one tenth of the scatter points. Similarly, we found that one-tenth of patients whose FFA/HDL-C $>0.75$ had a worse prognosis than the others, as shown in Figure 2. Interestingly, these scatter points may play an important role in predicting tumour grade, size, LNM and poor prognosis.

Although the patient numbers of FFAs $\geq 0.52 \mathrm{mmol} / \mathrm{L}$ and HDL-C $\leq 1.0 \mathrm{mmol} / \mathrm{L}$ were low, our results and their combined detection were credible. Firstly, the Surveillance, Epidemiology and End Results (SEER) database indicates that the incidence of NETs has increased significantly, approximately 5 times, to $5.25 / 100.000$ cases/year; of these, colorectal NETs account for approximately $49.6 \%$ of primary NET sites in the digestive tract[2]. Secondly, it is worth noting that patients who had FFA $\geq 0.52 \mathrm{mmol} / \mathrm{L}$ accounted for almost half of the total colorectal patients, and those with HDL-C $\leq 1.0 \mathrm{mmol} / \mathrm{L}$ accounted for almost one-fifth of the total; interestingly, patients with FFA $\geq 0.52 \mathrm{mmol} / \mathrm{L}$ and $\mathrm{HDL}-\mathrm{C} \leq 1.0 \mathrm{mmol} / \mathrm{L}$ also made up one-fifth of the patients with $F F A \geq 0.52 \mathrm{mmol} / \mathrm{L}$.

Traditionally, the level of FFAs has been detected to assess lipid metabolism and has been associated with hypertension, cardiovascular disease, type 2 diabetes, and obesity[17].Similarly, HDL-C, LDL-C, TG, CHOL, ApoA1 and ApoB, all widely used, were analysed to assess lipid metabolism in the body as combined biochemical indicators. Recently, an increasing number of researchers have indicated that FFAs and HDL-C are involved in colorectal cancer. FFAs are overexpressed in colorectal cancer [9], dietary palmitic acid

Page 15/22 
promotes cancer metastasis [18], and the reprogramming of fatty acid metabolism plays an important role in LNM of various cancers by the fatty acid-binding protein 5 (FABP5) pathway $[10,11]$.Interestingly, LNM and poor prognosis were significantly associated with the pre-treatment FFA level of colorectal NETs, consistent with our results. In addition, oxidative modification of HDL results in compositional and functional changes, and following increased cholesterol ester transfer protein (CETP) activity in parallel with decreased lecithincholesterol acyltransferase (LCAT) activity, HDL particles become larger, and changes in HDL composition, such as enrichment with TG and reduced ApoA1, paraoxonase-1 (PON1) and apoM and increased serum amyloid A (SAA) proteins, occur. The interaction of overexpressed SAA with TLR2 in cancer cells leads to cancer progression throughthe NF-KB-mediated pathway [19]. In addition, HDL-C was related to poor prognosis in patients with colorectal cancer, and very low levels of HDL-C ( $<30 \mathrm{mg} / \mathrm{dL})$ in women were significantly associated with cancer mortality $[13,14]$. In our study, tumour size $(\geq 2 \mathrm{~cm})$ was related to pretreatment HDL-C level, and worse OS was found in colorectal NETs with larger tumour size, consistent with the above researchers.

CgA (chromogranin A), synaptophysin (Syn) and CD56 are three neuroendocrine differentiation (NED) immunohistochemistry markers frequently used in NETs [20-22]. The results of immunohistochemistry are usually marked by semiquantitative scores to show positive cell percentages and positive cell staining intensities but are limited to qualification and by pathologist experience. Reports of CgA, Syn and CD56 are difficult to standardize, and it is difficult to predict prognosis by immunohistochemistry results directly. $\mathrm{CgA}$ in serum is an important biomarker in advanced pancreatic cancer and metastatic neuroendocrine tumours[23, 24], but due to the lower incidence of NETs[25] and the high cost of detecting reagents, the serological CgA test has not been widely performed. FFA and HDL-C are common biochemical biomarkers detected in clinical laboratories, and they have promising applications in predicting LNM and tumour size and predicting the OS of colorectal NETs.

The median survival and 5-year survival of patients also consistent with our conclusion. The median survival of patients with $\mathrm{FFA} \geq 0.52 \mathrm{mmol} / \mathrm{L}$ was $67.8 \pm 7.3$ months, the median survival of those with $\mathrm{HDL}-\mathrm{C} \leq 1.0$ $\mathrm{mmol} / \mathrm{L}$ was $53.8 \pm 9.7$ months, and the median survival of those with FFA $\geq 0.52 \mathrm{mmol} / \mathrm{L}$ and $\mathrm{HDL}-\mathrm{C} \leq 1.0$ $\mathrm{mmol} / \mathrm{L}$ was only $45.9 \pm 14.3$ months. The 5 -year survival of patients with FFA $\geq 0.52 \mathrm{mmol} / \mathrm{L}$ was $50.9 \%$, that of patients with $\mathrm{HDL}-\mathrm{C} \leq 1.0 \mathrm{mmol} / \mathrm{L}$ was $47.4 \%$ and that of patients with $\mathrm{FFA} \geq 0.52 \mathrm{mmol} / \mathrm{L}$ in combination with HDL-C $\leq 1.0 \mathrm{mmol} / \mathrm{L}$ was only $40.0 \%$. Therefore, patients with high FFA and low HDL-C had a worse prognosis than patients with only high FFA, only low HDL-C and patients with low FFA or high HDL-C. FFA/HDL-C predicted an even worse prognosis in terms of OS.

Mixed adenoneuroendocrine carcinoma of the colon and rectum are rare cancers; they are characterized by the presence of a combination of epithelial and neuroendocrine elements, where each component represents at least $30 \%$ of the tumour[26] and are unmet areas where NETs need to be described and defined[27]. In our study, there were more colorectal NET patents with positive LNM than with negative LNM, which may be due to not excluding mixed adenoneuroendocrine carcinoma among the patient groups, which is composed of poorly differentiated neuroendocrine carcinoma and easily metastasizes[26, 28].

Reduced plasma levels of HDL-C are a hallmark of obesity and cardiovascular diseases (CVDs); similarly, reduced ApoA1 has also been associated with cardiovascular risk[29]. Due to component differences between 
ApoA1 and HDL-C, a similar association was not found for LNM, tumour size or poor survival with HDL-C. However, the level of ApoA1 in colorectal NETs was significantly lower than that in controls, and the area under the receiver operating characteristic (ROC) curve was $82.2 \%$. Interestingly, the potential diagnostic ability is worth analysing. In addition, no significant association with pathological characteristics was found in $\mathrm{CHOL}$ and $\mathrm{ApoB}$ in our study.

There were some limitations in our study. First, it was of a retrospective design and included are relatively small number of patients. However, we believe the results are reliable. Because this study lasted more than 130 months, we could investigate the long-term survival outcomes and prognostic factors after different treatments, even with the small number of patients. Second, progression-free survival (PFS) data were not collected, and prognostic results could not be predicted comprehensively. Finally, further studies should be performed to validate our main conclusions.

\section{Conclusions}

FFA, CHOL, and ApoB were overexpressed in colorectal NETs, and HDL-C and ApoA1 were underexpressed. FFA $\geq 0.52 \mathrm{mml} / \mathrm{L}$ can be considered a cut-off point to predict LNM and poor OS, HDL-C $\leq 1.0 \mathrm{mmol} / \mathrm{L}$ can be considered a cut-off point to predict tumour size $\geq 2 \mathrm{~cm}$ and poor $0 \mathrm{~S}$, and FFA/HDL-C $>0.75$ can be considered another cut-off point to predict tumour grade, tumour size, LNM and poor OS.

\section{Abrreviation}

FFA: free fatty acid; HDL-C: How-density lipoprotein cholesterol; TG: triglycerides; CHOL: cholesterol; LDL-C: low-density lipoprotein cholesterol; ApoA1: apolipoprotein A1; ApoB: apolipoprotein B; OS: overall survival; PFS: progression-free survival; ROC: receiver operating characteristic; NETs: neuroendocrine tumours; SEER: Surveillance, Epidemiology and End Results; LNM: lymph node metastasis; CEA: carcinoembryonic antigen; CA19-9: carbohydrate antigen 19-9; CgA: chromogranin A; Syn: synaptophysin; FABP5: fatty acid-binding protein 5; CETP: Cholesterol ester transfer protein; LCAT: lecithin-cholesterol acyltransferase; PON1: paraoxonase-1 ; SAA: serum amyloid $A$.

\section{Declarations}

\section{Authors' contributions}

Bin Zhu, Haobo Huang and Yingping Cao wrote and edited the manuscript; Yingping Cao provided study material; Bin Zhu, Dan Wu, Pingli Yu, Haobo Huang, Haiyan Cai and Yuanyuan Yang analysed and interpreted the data; all authors reviewed and approved the manuscript.

\section{Acknowledgements}

Not applicable.

\section{Funding}


This work was supported by Start-up Fund for Scientific Research of Fujian Medical University (No. 2019QH1038 and 2019QH1046), the Joint Funds for the Innovation of Science and Technology in Fujian Province (No. 2019Y9050) and Natural Science Foundation of Fujian province (No. 2021J01738).

\section{Availability of data and materials}

All data generated or analyzed during this study are included in this published article. The datasets used and/or analyzed during the current study are available from the corresponding author on reasonable request.

\section{Ethics approval and informed consent to participate}

The present study was approved by the Ethics Committee of Fujian Medical University Union Hospital (2021 KJCX007) and was conducted in accordance with the Declaration of Helsinki. The written informed consents from all patients and/or their legal guardians were obtained and confirmed by the ethics committee of Fujian Medical University Union.

\section{Consent for publication}

Not applicable

\section{Competing interests}

The authors declare that they have no competing interests.

\section{References}

1. Yao JC, Hassan M, Phan A, Dagohoy C, Leary C, Mares JE, Abdalla EK, Fleming JB, Vauthey JN, Rashid A et al: One hundred years after "carcinoid": epidemiology of and prognostic factors for neuroendocrine tumors in 35,825 cases in the United States. Journal of clinical oncology : official journal of the American Society of Clinical Oncology 2008, 26(18):3063-3072.

2. Lawrence B, Gustafsson BI, Chan A, Svejda B, Kidd M, Modlin IM: The epidemiology of gastroenteropancreatic neuroendocrine tumors. Endocrinology and metabolism clinics of North America 2011, 40(1):1-18, vii.

3. Modlin IM, Lye KD, Kidd M: A 5-decade analysis of 13,715 carcinoid tumors. Cancer 2003, 97(4):934-959.

4. Frilling A, Akerström G, Falconi M, Pavel M, Ramos J, Kidd M, Modlin IM: Neuroendocrine tumor disease: an evolving landscape. Endocrine-related cancer 2012, 19(5):R163-185.

5. Dasari A, Shen C, Halperin D, Zhao B, Zhou S, Xu Y, Shih T, Yao JC: Trends in the Incidence, Prevalence, and Survival Outcomes in Patients With Neuroendocrine Tumors in the United States. JAMA oncology 2017, 3(10):1335-1342.

6. Bertani E, Ravizza D, Milione M, Massironi S, Grana CM, Zerini D, Piccioli AN, Spinoglio G, Fazio N: Neuroendocrine neoplasms of rectum: A management update. Cancer treatment reviews 2018, 66:45-55.

7. Ramage JK, De Herder WW, Delle Fave G, Ferolla P, Ferone D, Ito T, Ruszniewski P, Sundin A, Weber W, Zheng-Pei Z et al: ENETS Consensus Guidelines Update for Colorectal Neuroendocrine Neoplasms. 
Neuroendocrinology 2016, 103(2):139-143.

8. Stich V, Berlan M: Physiological regulation of NEFA availability: lipolysis pathway. Proc Nutr Soc 2004, 63(2):369-374.

9. Zhu B, Zhang J, Zheng Q, Dong B, Wang M, Liu J, Cao Y: Free Fatty Acid is a Promising Biomarker in Triage Screening for Patients with Colorectal Cancer: A Case-Control Study. Cancer management and research 2021, 13:3749-3759.

10. Zhang C, Liao Y, Liu P, Du Q, Liang Y, Ooi S, Qin S, He S, Yao S, Wang W: FABP5 promotes lymph node metastasis in cervical cancer by reprogramming fatty acid metabolism. Theranostics 2020, 10(15):65616580.

11. Kawaguchi K, Senga S, Kubota C, Kawamura Y, Ke Y, Fujii H: High expression of Fatty Acid-Binding Protein 5 promotes cell growth and metastatic potential of colorectal cancer cells. FEBS Open Bio 2016, 6(3):190-199.

12. Ganjali S, Ricciuti B, Pirro M, Butler AE, Atkin SL, Banach M, Sahebkar A: High-Density Lipoprotein Components and Functionality in Cancer: State-of-the-Art. Trends in endocrinology and metabolism: TEM 2019, 30(1):12-24.

13. Wang Y, Sun XQ, Lin HC, Wang DS, Wang ZQ, Shao Q, Wang FH, Yan SM, Liang JY, Zeng ZL et al: Correlation between immune signature and high-density lipoprotein cholesterol level in stage II/III colorectal cancer. Cancer Med 2019, 8(3):1209-1217.

14. Penson P, Long DL, Howard G, Howard VJ, Jones SR, Martin SS, Mikhailidis DP, Muntner P, Rizzo M, Rader DJ et al: Associations between cardiovascular disease, cancer, and very low high-density lipoprotein cholesterol in the REasons for Geographical and Racial Differences in Stroke (REGARDS) study. Cardiovascular research 2019, 115(1):204-212.

15. Bosman F CF, Hruban R, Theise N, eds: World Health Organization Classification of Tumours of the Digestive System. Lyon, France: IARC Press; 2010.

16. Klimstra DS, Modlin IR, Coppola D, Lloyd RV, Suster S: The pathologic classification of neuroendocrine tumors: a review of nomenclature, grading, and staging systems. Pancreas 2010, 39(6):707-712.

17. Ghosh A, Gao L, Thakur A, Siu PM, Lai CWK: Role of free fatty acids in endothelial dysfunction. Journal of biomedical science 2017, 24(1):50.

18. Pascual G, Domínguez D, Elosúa-Bayes M, Beckedorff F, Laudanna C, Bigas C, Douillet D, Greco C, Symeonidi A, Hernández I et al: Dietary palmitic acid promotes a prometastatic memory via Schwann cells. Nature 2021.

19. Ganjali S, Banach M, Pirro M, Fras Z, Sahebkar A: HDL and cancer - causality still needs to be confirmed? Update 2020. Seminars in cancer biology 2021, 73:169-177.

20. Maclntosh PW, Jakobiec FA, Stagner AM, Gilani S, Fay A: High grade neuroendocrine neoplasm of the antrum and orbit. Survey of ophthalmology 2015, 60(5):486-494.

21. Modlin IM, Gustafsson BI, Moss SF, Pavel M, Tsolakis AV, Kidd M: Chromogranin A-biological function and clinical utility in neuro endocrine tumor disease. Annals of surgical oncology 2010, 17(9):2427-2443.

22. Wiedenmann B: Synaptophysin. A widespread constituent of small neuroendocrine vesicles and a new tool in tumor diagnosis. Acta oncologica (Stockholm, Sweden) 1991, 30(4):435-440. 
23. Malaguarnera M, Cristaldi E, Cammalleri L, Colonna V, Lipari H, Capici A, Cavallaro A, Beretta M, Alessandria I, Luca $S$ et al: Elevated chromogranin $A(\mathrm{CgA})$ serum levels in the patients with advanced pancreatic cancer. Archives of gerontology and geriatrics 2009, 48(2):213-217.

24. Warner RR, Curran T, Shafir MK, Schiano TD, Khaitova V, Kim MK: Serum and ascites chromogranin-A in patients with metastatic neuroendocrine tumors. Pancreas 2011, 40(4):622-626.

25. Cives M, Strosberg JR: Gastroenteropancreatic Neuroendocrine Tumors. CA Cancer J Clin 2018, 68(6):471-487.

26. Qiu S, Pellino G, Warren OJ, Mills S, Goldin R, Kontovounisios C, Tekkis PP: Mixed adenoneuroendocrine carcinoma of the colon and rectum. Acta chirurgica Belgica 2018, 118(5):273-277.

27. Ramage JK, Valle JW, Nieveen van Dijkum EJM, Sundin A, Pascher A, Couvelard A, Kloeppel G: Colorectal Neuroendocrine Neoplasms: Areas of Unmet Need. Neuroendocrinology 2019, 108(1):45-53.

28. Milione M, Maisonneuve P, Pellegrinelli A, Grillo F, Albarello L, Spaggiari P, Vanoli A, Tagliabue G, Pisa E, Messerini $L$ et al: Ki67 proliferative index of the neuroendocrine component drives MANEC prognosis. Endocrine-related cancer 2018, 25(5):583-593.

29. Andrikoula M, McDowell IF: The contribution of ApoB and ApoA1 measurements to cardiovascular risk assessment. Diabetes, obesity \& metabolism 2008, 10(4):271-278.

\section{Figures}
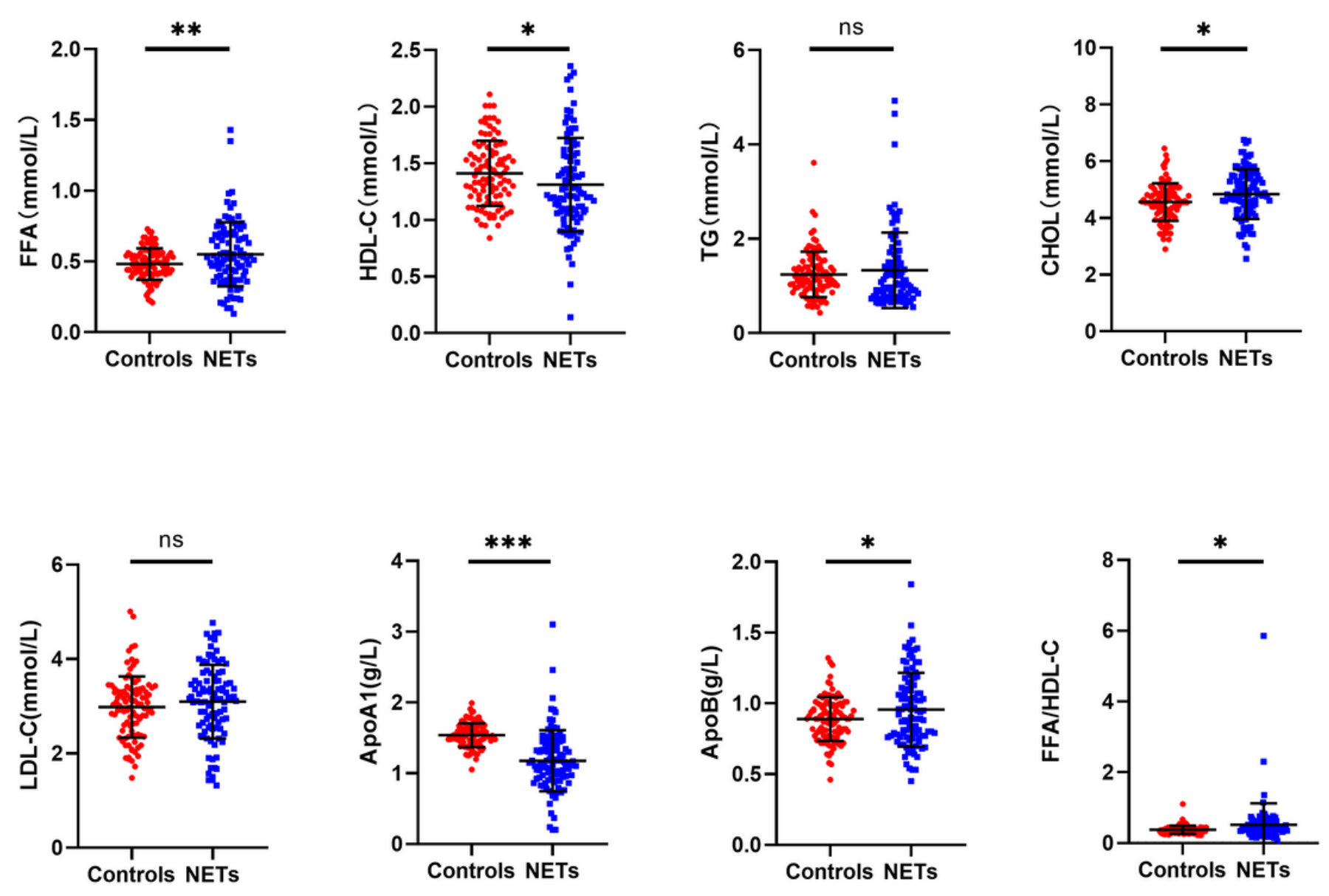


\section{Figure 1}

The pre-treatment levels of FFA, HDL-C, TG, CHOL, ApoA1, LDL-C and ApoB in controls and patients with colorectal NETs.

Controls: Normal persons were matched by age and sex to patients with colorectal NETs one by one. Abbreviations: FFA, free fatty acid; HDL-C, How-density lipoprotein cholesterol; TG, triglycerides; $\mathrm{CHOL}$ : cholesterol, LDL-C, low-density lipoprotein cholesterol; ApoA1, apolipoprotein A1; ApoB, apolipoprotein B.



\section{Figure 2}

Kaplan-Meier survival curves for colorectal NET patients with different tumour grades, tumour sizes and lymph node metastases, as well as with respect to pre-treatment FFA, HDL-C and FFA/HDL-C levels in serum; the relationship between 5-year survival and FFA/HDL-C level was also drawn above. 